sich die "Zunft« kleinkariertes Mauern. Methodische Orientierung jenseits der dahinterstehenden politischen Inhalte und Gruppeninteressen ist (noch) kein Thema, kein allgemein akzeptiertes Kriterium für Professorenmärkte, stößt demgemäß in Studium und Examen auf Akzeptanzprobleme.

Die Frage wäre also, ob Marktorientierung das Weitertreiben oder Realisieren der steckengebliebenen, der »verweigerten « Ausbildungsreform ermöglichen kann. Modellhaft exerzierte's Ausbildungsdauer oder Europa 1992/93 sind dabei als Konsenserzielungsstrategien schnell zu entlarven.

Am Start der Versuchsstrecke steht, an dieser Klarstellung liegt mir, die Tauglichkeit funktionaler Äquivalente zur administrativ verordneten Juristenausbildung. Staatsfreiheit möchte ich gerne, optimierte Elitebildungen dagegen nicht als das Maß der Dinge akzeptieren.

\title{
Rüdiger Lautmann \\ Vom Wildwuchs zum Bonsai - die Rechtssoziologie im Lehrbuch
}

Läßt Soziologie sich lehren? Ja. Läßt Soziologie sich aus einem Lehrbuch lernen? Nein. Muß es Lehrbücher für Rechtssoziologie geben? Wohl ja. Derlei Fragen und widersprüchliche Antworten plagen einen beim Lesen der langersehnten und vor drei Jahren auf einen Schlag erschienenen Gesamtdarstellungen zur Rechtssoziologie $^{1}$.

$I$.

Die Bücher - das sei gleich vorweg bemerkt, und ich werde es mehrfach wiederhoJen, um Licht und Schatten auszugleichen - erfüllen ihre deklarierten Zwecke ausgezeichnet: sie unterrichten zuverlässig über das, was derzeit als Rechtssoziologie betrieben und veröffentlicht wird. Zu diesen Titeln ist auch Manfred Rehbinders 1989 neubearbeitetes und zum Lehrbuch aufgewertetes Werk zu rechnen. Nicht aber ein Titel wie Luhmanns sehr bekannte »Rechtssoziologie" (3. Aufl. 1987, 385 S., Opladen: Westdeutscher Verlag); denn diese ‘ist< das, worüber die anderen meist nur reden.

Worin besteht der Unterschied zwischen Lehrbuch auf der einen, authentischer

is Im Modell stellt sıch der zeitliche Rahmen der derzeıt praktızıerten Jurıstenausbildung ausgesprochen überschaubar dar ( $31 / 2$ Jahre Studium $+2 \frac{1}{2}$ Jahre Referendarıat), unterschetdet die stattfindende also in nıchts von noch so schön reformierten Ausbildungen. Damıt soll nıcht der Konserverung eınes längst überfälligen Zustandes von Jurıstenausbildung das Wort geredet werden. Nur können die Gründe langer Ausbildungszetten nıcht enfach "wegmodelliert " werden. Ursächlich sind die notwendige und gesellschaftsadäquate Komplexıtät der Studienınhalte und die Bedingungen der Massenunıversıtät. Genau mıt diesen Verzögerungsfaktoren setzen die präsentserten Reformvorschläge sich meıst erst gar nıcht auseinander.

1 Klaus F. Röhl: Rechtssozıologıe. Ein Lehrbuch. Köln (Heymanns) 1987, $48+595$ S., Ln., 138,- DM. Thomas Raiser: Rechtssoziologie. Ein Lehrbuch. Frankfurt a. M. (Metzner) $1987,18+{ }_{364}$ S., brosch., 39,- DM.

Hubert Rottleuthner: Einführung in die Rechtssozıologıe. Darmstadt (Wiss. Buchges.) 1987, $11+200$ S., brosch., 44,- DM.

Manfred Rehbinder. Rechtssozıologıe, 2. Aufl. Berlin (de Gruyter) 1989, $8+267$ S., brosch., 38,- DM. 
Rechtssoziologie auf der anderen Seite? Hier muß das Janusgesicht einer sogenannten Bindestrich-Soziologie in Rechnung gestellt werden. Ein solches Fach hat nämlich zwei Aufgaben und damit zwei Blickrichtungen: zum einen soll es einen Gegenstandsbereich, hier das Recht, für die allgemeine Soziologie erschließen; zum anderen soll es die soziologische Sichtweise denjenigen eröffnen, die für den Gegenstandsbereich wissenschaftlich und praktisch tätig sind - hier also der Jurisprudenz in Wissenschaft und Berufen. Sehr bewußt stellen sich Raiser und Röhl der zweiten Aufgabe.

Zugespitzt: Soziologie beschreibt man nicht, man betreibt sie. Eine Vorführung der Soziologie, wie andere sie denken, macht so satt wie nacherzähltes Mittagessen.

Immerhin mag es den Appetit wecken. Tun das solche Bücher? Für manche der Leser/innen vielleicht; für die meisten indes ersparen sie den Griff zu den Originalen. Diese werden konsumierbar dargeboten - das ist der Sinn solcher Lehrbücher -, und die sschönen<, d.h. vielzitierten Stellen erscheinen sogar wörtlich zitiert.

Gut sind die rechtssoziologischen Lehrbücher immer dann, wenn sie zur Lektüre der originalen Schriften hinführen. Das gelingt, wenn sie deren Fragestellung und theoretischen Hintergrund erläutern, wenn sie - für die ja etwas entrückten Klassiker - die historisch-kritischen Anmerkungen liefern. Schlecht wären rechtssoziologische Lehrbücher, wenn sie Rechtssoziologie kanonisierten; also richtige Lesarten vorschrieben. Und lächerlich würden sie sich machen, wenn sie Noten erteilten: was Rechtssoziologie dürfe und solle, was hingegen nicht. Unsere Lehrbücher tun das nicht, obschon das Auftreten der Rechtssoziologie in den siebziger Jahren eine Fülle solcher Zensuren hervorgerufen hatte. Aber die Schlachten von damals sind geschlagen.

II.

Wenn der Friede geschlossen ist, spricht man nicht mehr vom Krieg. Und wie lautet doch gleich die Anstandsregel vom Hause des Gehenkten und dem Strick? Ich muß mich nicht daran halten und frage mich, warum wir plötzlich drei Lehrbücher der Rechtssoziologie bekommen haben. Daß sie erscheinen konnten, die Bedingung ihrer Möglichkeit sozusagen, hat mit dem Scheitern des Versuchs zu tun, Rechtswıssenschaft als Sozzalwissenschaft zu veranstalten. Vonseiten der Soziologie war damals versucht worden, Teile der juristischen Entscheidungstätigkeit für sich zu reklamieren. Diese reichlich vereinnahmende Liebe - ich selber habe sie großmäulig mitverkündet - wurde harsch zurückgewiesen.

Von den leidenschaftlichen Kanonaden liest man in den sich abgeklärt gebenden Büchern kaum etwas, obwohl die Verfasser durchaus inmitten des Getümmels gestanden haben. Raiser erläutert das Scheitern der Ausbildungsreform - mit ihr verbunden die Rechtssoziologie als Ausbildungsfach - mit dem Mißtrauen von »konservativ gesonnenen Hochschullehrern« (S. 28). Außerdem erzwang die gestiegene Zahl der Studierenden eine »Konzentration aller Kräfte auf die rechtsdogmatischen Hauptfächer«. Im Abschnitt über ,Rechtssoziologie und Rechtsdogmatik behandelt Raiser nicht etwa die unerlaubten Annäherungen, sondern das Gewohnheitsrecht und die Generalklauseln (S. 10). Auch die anderen Bücher sparen die Frage aus. Die Zurückhaltung wirkt vornehm und schont vernarbte Wunden. Doch dermaleinst wird der Zustand der Rechtssoziologie als Resultat der abgelaufenen Kämpfe und Niederlagen beschrieben und begriffen werden können.

Der damalige Anspruch ging viel weiter, als eine Spezialdisziplin Rechtssoziologie zu etablieren (obwohl dies gelungen und geblieben ist). Röhl sagt sehr zu Recht: »Eine verselbständigte Rechtssoziologie kann die Integration der Sozialwissenschaf- 
ten in die dogmatischen Fächer nicht ersetzen « (S. 2). Eine Soziologie des Rechts ist zu haben; viel schwieriger ist es für eine Soziologie $\imath m$ Recht. Kaum eine juristische Entscheidung - in der Wissenschaft, in der Beratung, in der Verwaltung - wird ausschließlich unter dem Gesichtspunkt gefällt, ob das Resultat normrichtig sei; immer sind auch ökonomische, politische, weltanschauliche und klientenbezogene Rücksichten im Spiel. Der rechtsdogmatisch abgeleitete Rahmen wird durch einige weitere Rationalitätskriterien aufgefüllt. Manche davon sind sozialwissenschaftlich konstituiert (auch wenn der Verwendungszusammenhang diese Herkunft vielleicht nicht mehr erkennen läßt). Wie verhalten sich die Bücher dazu?

Daß das Experiment der emphastgen Jurtstenausbildung (1971-1984) das Saatbeet neuerer Rechtssoziologie in der Bundesrepublik gewesen ist, wird in keinem dieser Bücher besprochen. Nur vordergründig läßt sich das so erklären, daß alle Autoren als Professoren in der klassischen Juristenausbildung arbeiten. Vielmehr scheint es, ats wäre es bereits zu spolitisch،, das überlebenwollende Fach Rechtssoziologie mit Beginn und Abbruch jener Reform in einem Atemzug zu nennen (Raiser deutet es an, S. 28). Der Wechsel von Politikphasen in der Bundesrepublik (1969, 1982) verantwortete sowohl Start wie Abbruch des Ausbildungsexperiments. So werden sich Leistungen und Blindflecken der Rechtssoziologie nur dann verstehen lassen, wenn ihre politisch-institutionelle Verflochtenheit mit in Betracht gezogen wird. Obgleich der Methodenstreit der siebziger Jahre heute fast so fern anmutet wie jener andere um 1910 über Freirecht, soziologische und Interessenjurisprudenz, bleibt Anlaß, sich beider zu erinnern. Hier wie da wurden Weichen für die Fahrt der Rechtswissenschaft i.e.S. und der Rechtssoziologie gestellt, und die gefundene Richtung bleibt für lange Zeit konstant.

III.

Nun aber in gutem Sinne zu den Lehrbüchern. Die hier schreibenden Rechtswissenschaftler sind keineswegs bloß raufgeschlossene Juristen`, wie E. Blankenburg und H. Treiber sanft ironisch die juristischen Liebhaber (Dilettanten) der Soziologie einmal benannt haben. Sie sind eindeutig Rechtssoziologen, mit breiten Kenntnissen und tiefgehendem Verständnis für die Soziologie. Röhl hält es für selbstverständlich, »ein Rechtsinstitut auch auf seine sozialen Voraussetzungen und gesellschaftlichen Auswirkungen hin zu befragen, ohne dabei die grundsätzlich gegenläufigen Funktionen von Soziologie und Dogmatik aus dem Auge zu verlieren« (S. 2).

Die Bücher sind für die gegenwärtige Rechtssoziologie ebenso repräsentativ wie ihre Autoren zur Darstellung des Stoffs berufen sind, arbeiten sie doch seit mehr oder weniger zwei Jahrzehnten auf dem Gebiet. Die gesammelten Recherchen und Reflexionen einer so langen Beschäftigung führen zu einer argumentativ runden, literarisch gesättigten und weithin gerecht gewichtenden Präsentationen rechtssoziologischer Resultater. Wer selber Rechtssoziologie lehrt und sich des Eingeständnisses seiner Hilfsbedürftigkeit nicht schämt, wird sich hierin immer wieder schlau machen können. Auch wenn mein Unbehagen bei der Lektüre nie verschwand, will ich gern bekennen, daß man es eigentlich kaum besser machen kann.

$\mathrm{Da}$ dies etwas pauschalierend klingt und als unaufrichtig mißverstanden werden könnte, will ich im folgenden die Bücher einzeln charakterisieren, wobei ähnliche Gesichtspunkte angelegt werden, um einen Vergleich zu ermöglichen. 
Das Lehrbuch von Röhl hebt sich in Umfang, Themenvielfalt und Literaturfülle aus allen heraus. Auch wenn er die makrosoziologischen und manchmal recht globalen Theorien über das Recht keineswegs ausspart, hält er es doch für gefährlich, mit großen Hypothesen zu arbeiten, und will sich mit "bescheideneren Aussagen begnügen " (S. 33). Damit zielt er auf die (auch bei den anderen favorisierten) "Theorien mittlerer Reichweite« (S. 52 f.).

Bei Röhl finden sich die meisten Ausführungen über allgemein-soziologische Theoreme. Ja, er baut sein Buch im wesentlichen nach den Theorieschulen der Soziologie auf (S. 1 19-524). Damit überwindet er auch den Gegensatz von Soziologie im Recht und Soziologie des Rechts; er ordnet die Themen der Rechtssoziologie jeweils einer Richtung der Allgemeinen Soziologie zu. Der Preis dafür - auch die anderen Bücher entrichten ihn - ist ein gewisser Eindruck von Eklektizismus. Bei Röhl äußert sich das, beispielsweise zur Konkurrenz zwischen methodologischem Individualismus und dto. Kollektivismus, so: "Je nach der Art der Sachprobleme, die man behandeln will, kann es zweckmäßig sein, von der einen oder anderen Seite her, oder auch von beiden anzusetzen (S. 129).

In erstaunlichem Maße gelingt es Röhl, rechtssoziologische und sonstige sozialwissenschaftliche Resultate mit Problemen aus der Rechtsdogmatik und -praxis zu verknüpfen. Dazu werden zahllose Beispiele gebracht, die sowohl juristisch wie soziologisch ausführlich erläutert und darüber hinaus noch gut dokumentiert sind.

Zu charakterisieren ist >der Röhk als Großlehrbuch, als ein auf Vollständigkeit bedachtes Nachschlagewerk. Er ist auf breitestmögliche Literaturverwendung bedacht (der Index listet an die tausend Namen auf). Geeignet ist er zur Lektüre als 'klassisches Lehrbuch`, wie in der Rechtswissenschaft als literarisch höchstrangige Gattung etabliert.

\section{V.}

Rasser faßt sich knapper, zielt aber gleichfalls auf umfassende Information über alle in der Rechtssoziologie heute wie früher erörterten Fragen. Schön sind die kritischen Porträts von sieben Theoretikern des Fachs (S. 33-139).

Es gelingt Raiser an zahllosen Stellen, die soziologischen und rechtswissenschaftlichen Theoreme in Kommunikation zu bringen: sei es auf sprachlich-begrifflicher Ebene, sei es im historischen Ablauf, sei es zur Kausalerklärung juristischer Phänomene, sei es als Angebot zur Auslegung, Ermessensausübung und Lückenfüllung beim juristischen Entscheiden. Gewissermaßen nach dem Prinzip des Reißverschlusses vereinigen sich die beiden Denkströme.

Die Grenze zwischen den Disziplinen wird etwas deutlicher gezogen als bei Röhl. In seiner Würdigung Durkheims scheint Raiser mitzuteilen, die »Verbindung von Wissenschaft und Moral bzw. Wissenschaft und Praxis « sei problematisch. Ein Versprechen der Wissenschaft, »krankhafte Zustände aufzudecken und die Wege zu ihrer.Heilung zu weisen «, sei »heute ungebrochen nicht mehr nachvollziehbar « (S. 56). Vielmehr gehe jeder Forscher von Wertungen aus und müsse dies deutlich machen. Damit verkürzt sich natürlich ganz entscheidend die Kompetenz der Soziologie, zu den in juristischen Konflikten symptomatisch erscheinenden gesellschaftlichen Mißständen etwas intersubjektiv Verläßliches beizutragen.

Eine Besonderheit bildet Raisers Schlußteil: »Das rechtssoziologische Portrait der Bundesrepublik (S. 3 13-348). Unter dem Gesichtspunkt der Rechtskultur werden 
die Merkmale des hiesigen Staatswesens vergleichend und politisch sensibel beschrieben.

$\mathrm{Zu}$ charakterisieren ist der Raiser als Lehrbuch im Sinne eines Studienbuchs. Verweise auf grundlegende und weiterführende Literatur werden umfangreich gegeben. Zur gezielten Schnellinformation eignet sich das Werk ebenso wie zur Prüfungsvorbereitung - im positiven Sinne also ein Lehrbuch.

$V I$.

Rebbınder hat seinen Göschen-Grundriß nach zwölf Jahren, bei der Übernahme als "de Gruyter Lehrbuch", stark erweitert. Der Autor berücksichtigt die drei anderen hier besprochenen Bücher und gibt entsprechende Verweise. Einerseits ist dieses Buch das jüngste, andererseits indessen in weiten Teilen der Entstehung seiner ersten Auflage zur Mitte der siebziger Jahre verpflichtet. Einschlägige Forschungen aus der Schweiz werden eigentlich nur hier umfassend berücksichtigt; insoweit kann Rehbinder neben dem an sich >vollständigenء Röhl mit Gewinn zu Rate gezogen werden.

Unter unseren Autoren ist Rehbinder derjenige, der am längsten in der Rechtssoziologie aktiv ist - seit bald dreißig Jahren. Weniger als andere spart er rechtspolitische Wertungen und Empfehlungen aus; in seinen neugeschriebenen Kapiteln, etwa zur Gesetzgebung (S. 233-249), findet sich manch temperamentvolle Kritik. Mehrfach wird das Buch zum Essay, was dem Fluß und Vergnügen des Lesens nur entgegenkommt.

Rehbinder stützt sich insbesondere auf die ältere Rechtssoziologie sowie auf zahlreiche Arbeiten seiner Schüler. Das Werk eignet sich zum Kennenlernen der rechtssoziologischen Tradition und eines sich darauf gründenden Denkens, das gegen Moden resistent ist. Wir haben es hier mit einem eigenständigen rechtssoziologischen Denkstil zu tun; Rehbinder stand einmal der Soziologie sehr nahe.

\section{$V I I$.}

Das Buch von Rottleuthner zieht stilistisch und inhaltlich einen scharfen Kontrast zu den anderen. Die vielen Ironien über die Spiele der Wissenschaft (einige Beispiele: S. 7, 92, 174) bezeugen eine prinzipiell kritische Haltung, wie sie den anderen Autoren nicht eignet. Für den Autor ist es wichtig, die kleinen Gewißheiten der vorhandenen Rechtssoziologie erneut darauf zu befragen, ob sie denn auch stimmen. Streng empiristisch betrachtet er die Stichproben, Korrelationen und Kausalinterpretationen - und findet viel Lückenhaftes, Überfolgertes und Falsches. Liebgewordene Deutungen werden brüchig oder hinfällig. Dieses gilt etwa

- zum Einfluß organisations- und konfliktfähiger Interessen auf den Inhalt der Gesetze (S. 50);

- zur Ziviljustiz als Dienstleistungsbetrieb für die Geschäftswelt (S. 1 16-I I 8);

- zum Zusammenhang zwischen parteipolitischem Hintergrund und richterlichem

Entscheidungsverhalten (S. 106-1 10);

- zur Selektivität der Strafverfolgung (S. 137, 177) sowie

- zur Bevölkerungsmeinung hinsichtlich des Rechts (S. 174).

Rottleuthner will "eine hochabstrakte Theoriediskussion auf die Ebene empirischer Überprüfbarkeit ziehen" (S. 55 f.). Das tut er denn auch mit eigenen Studien (beispielsweise S. 4 I f., $60 \mathrm{ff}$., 6 fff., $106 \mathrm{ff}$., II 4 ff.). Wenn Rottleuthner die empirische Sozialforschung so ernsthaft betreibt, bleibt er in gewisser Weise seinem früheren $\mathrm{Spa}$ an der Methodologie durchaus treu. 
Sozialtheoretisch scheint er (neuerdings) verhaltens- und systemtheoretischen Ansätzen zuzuneigen. Makrosoziale Determinanten werden abgelehnt (etwa in der Gesetzgebungstheorie, S.46). Positionen des methodologischen Individualismus werden hervorgehoben (etwa das utilitaristische Handlungsmodell von K.-D. Opp und A. Diekmann, S. s6-63).

Im Hintergrund steht immer der Anspruch, eine neue Stufe der Rechtssoziologie zu markieren, indem das neuere Wissen systematisiert wird (S. r). Das Unterfangen ist m. E. schwierig, weil die empirischen Resultate selber nicht systematisch generiert sind. Oder gab es einen geheimen Forschungsplan der Rechtssoziologie?

Soziologisches und sozialphilosophisches Denken, wie es uns hier authentisch entgegentritt, kann das Gesellschaftliche nicht als etwas Festes, Gegebenes ansehen, sondern als einen Fluß von Problemen, die sich ständig erneuern, so daß keine 'Lösung ihnen gewachsen ist. Ein Kompendium svorläufiger Endergebnisse der Rechtssoziologie läßt sich danach kaum noch denken.

Unter Ausbildungsgesichtspunkten eignet sich Rottleuthners Einführung für Seminare und zur Projektvorbereitung. Manche Ergebnisse empirischer Vorhaben werden hier erstmals mitgeteilt. Man sollte das Buch stets neben und ergänzend zu einem der anderen heranziehen.

VIII.

Nachdem die Bücher einzeln gewürdigt sind, will ich für einige ausgewählte Gesichtspunkte einen Vergleich anstellen, der jeweils durch alle vier führt.

Klassiker? Einmütig werden Emile Durkheim und Max Weber in den Vordergrund gerückt. Auch Eugen Ehrlich erhält einige Ehrbezeugung sowie bei einzelnen Lehrbuchverfassern manch anderer Autor. Jedoch wird jeder der >Klassiker unterschiedlich akzentuiert. So findet sich Durkheim von Röhl in einem eigenen Kapitel Werk für Werk dargestellt (S. 20-26, I 27-1 29); später wird der Name oft erwähnt, wenn ein entsprechender Begriff auftaucht, wie etwa >Restitution<. Auch Raiser stellt Durkheim dar (S.46-57), relativiert aber den Sendungsanspruch. Rottleuthner verweigert Durkheim den Status des Rechtssoziologen; dieser habe das Recht nur methodisch benutzt (S. 1 8-2 r).

Klassenjustiz? Die juristischen Autoren lehnen die damit verbundenen Vorstellungen als zu generalisierend $\mathrm{ab}$. Die Resultate empirischer Untersuchungen über die Selektivität rechtlicher Verfahren, Zugangsbarrieren, Verzerrungen beim Entscheiden werden zuverlässig berichtet. Sie stützen in der Tat kein Pauschalurteil, ohne daß sämtliche Fragen als beantwortet gelten könnten. Rottleuthner benennt denn auch stets die Forschungsdesiderate.

Gesellschaftspolitischer Standort. Linke schreiben keine Lehrbücher, so möchte ich vermuten. Die in der Kritischen Justiz typischerweise vertretenen Positionen werden in den Texten zur Kenntnis genommen, aber nicht gerade hervorgehoben. Doch keineswegs stehen die besprochenen Autoren >rechts<, in welchem Sinne immer. Etablierte Themen der Gesellschafts- und Rechtskritik werden hier ausdrücklich geteilt; die Autoren argumentieren etwa

- für die Gleichstellung der Frauen;

- gegen die Diskriminierung von Rassen und Randgruppen;

- für die Kompensation sozialer Benachteiligungen durch Verfahrenshilfen;

- für die Verfolgung von Wirtschafts- und Umweltvergehen.

Der Mut und die Leistungen der Protestbewegungen und Bürgerinitiativen werden anerkannt. Gesellschafts- und wissenschaftspolitische Konflikte, in denen einige 
Autoren in rechtssoziologisch spannungsgeladeneren Jahren durchaus gestanden haben, kommen in den Büchern allerdings nicht zum Ausdruck. Nur an der Literaturauswahl und -kommentierung sind eigene Stellungnahmen gelegentlich blaß erkennbar. Das politische Ideal der heutigen Rechtssoziologie ist eindeutig und ohne jeden Abstrich »der liberale und soziale Rechtsstaat" (in den Worten von Raiser, S. IV und 348).

Wissenschaftstdeal. Auch hier zeigt sich eine etwas überraschende Einhelligkeit. Zwar werden meta-ethische bzw. werturteilsbezogene Probleme zunächst einmal umschifft, indem sie in neutraler Haltung klassifiziert werden: etwa als theoretische, empirische oder kritische Rechtssoziologie (Raiser, S. 8). Doch das Credo bleibt nicht vorenthalten: "Die wirklichkeitswissenschaftliche Betrachtung gelangt begriffsnotwendig an ihre Grenze, sobald die Frage aufgeworfen wird, ob die vorgefundene Ordnung inhaltlich auch richtig im Sinn von human, gut, billig, gerecht usw. sei, und ob sie als solche festzuhalten sei oder der Verbesserung bedürfe« (S. rof.).

Anklänge an den Logischen Empirismus bzw. Kritischen Rationalismus sind in den Büchern allenthalben zu vernehmen (vgl. Röhl, S. 83-86, Rehbinder, S. 69). Empirie wird weit hochgehalten. Röhl will Rechtssoziologie als den Versuch verstanden wissen, wallein mit empirischen Methoden dem Verhältnis von Recht und Gesellschaft auf den Grund zu gehen« (S. 7r). Das ist nur zu verständlich; denn allein mit betontem Empirismus kann Soziologie die Wirklichkeit nach ihren eigenen Konzeptionen interpretieren, d.h. die konkurrierenden Interpretationen der Jurisprudenz konterkarieren.

Fachidentttät. Wir sehen, wie einheitlich unsere Rechtssoziologie in Grund- und Einzelfragen verfährt. (Die Reihe der Vergleichspunkte ließe sich fortsetzen: etwa zur Justizforschung, zur angeblichen Prozeßflut, zur Gesetzesflut.) Die Identität als Forschungsdisziplin scheint in hohem Maße gegeben - ein durchaus überraschendes Ergebnis. Die Soziologie ist ja eine ihrer Fachidentität weithin unsichere Wissenschaft, weil szu jung und krisengeschüttelt, weil zu sehr von anderen Grundlagenfächern abgeleitet, weil ,Nebenfach par excellence usw. In unserem Fall demonstrieren versierte Juristen, selber die Zweifel am Wissenschaftscharakter ihres eigenen Fachs souverän beiseitewischend (vgl. Röhl, S. 66), wie eine richtige, d. h. selbstgewisse Fachdisziplin sich präsentiert: mit ihrer Geschichte, mit sihren< Methoden und ihren Theoremen. Sozusagen: als Denkkomplex, den es immer gab, immer geben wird, einfach geben muß.

Die Fachidentität wird in den Büchern auf unterschiedliche Weise hergestellt, wobei die Grenze zur Rechtswissenschaft i. e.S. betont wird. Dazu Röhl: „Die Rechtssoziologie dagegen versteht sich als eine alle Erscheinungen des Rechts umfassende sozialwissenschaftliche Disziplin mit eigenen Erkenntnisinteressen, die sich ihre Themen nicht vom Recht und den Juristen vorgeben läß« (S. 49). Und nochmals: »Die Rechtssoziologie ist nicht als juristische Hilfsdisziplin angetreten, sondern als eine Wissenschaft mit eigenen Erkenntniszielen « (S. 87). Das Selbstverständnis wird auch durch den Verweis auf die internationale Fachgemeinschaft fundiert $($ S. 57,63$)$. Raiser gründet die Fachidentität auf erkenntnistheoretische Abgrenzungen (S. 3-14), Rehbinder auf die Eigenständigkeit von Forschungsbereich und Arbeitsinstrumenten (S. I-89).

Stutzen läßt allenfalls die Aussage, von der Soziologie her betrieben habe die Rechtssoziologie ein anderes Erkenntnisinteresse als von der Jurisprudenz her gesehen (Rehbinder, S. 5 f.). Diese Feststellung ist realistisch, kommt aber in den anderen Büchern nicht zum Ausdruck - erst die Einigkeit macht Schwache stark. Rottleuthner sieht die Fachidentität noch als unterwegs. Die institutionelle Absiche- 
rung ist »nur begrenzt gelungen «, und »die soziologische Zunft bleibt... eigenartig rechtsfremd « (S. . ).

$I X$.

In den Wein des Einigseins gießen meine abschließenden Überlegungen einige Wermutstropfen. Wie gut vereinbaren sich der Typ des juristischen Lehrbuchs und die soziologische Rechtsanalyse? Die Konvergenz unter den drei juristischen Autoren ist so groß bzw. deren Integration in eine gemeinsame Wissenschafts- und Lehrbuchkultur so überzeugend, daß es erlaubt sei, sie gemeinsam dem Buch von Rottleuthner gegenüberzustellen.

Was ist 'beim Soziologen anders? Etwa dies: je herrschender eine Meinung, desto lieber verstößt er dagegen. Beispielsweise läßt Rottleuthner die Geschichte der Rechtssoziologie mit Montesquieu beginnen (S. 7-II) statt, wie die anderen, bei Marx/Durkheim/Weber. Für manche sonst von Buch zu Buch weitergereichte Hauptthese klagt Rottleuthner die (mangelnde) empirische Fundierung ein; so zur angeblichen Rematerialisierung der Gesetze (S. SI). Die Großen Texte werden von ihm gern gegen den Strich gelesen, um ihnen genüßlich nachzuweisen, wo sie sich täuschen, wo sie sich über Schwierigkeiten hinwegmogeln (etwa zu Durkheim auf S. I 8, 21). Solche Unterschiede sind keineswegs bloß subjektiv und temperamentsbedingt; sie verweisen auf eine Verschiedenheit der Denkstile.

Wenn die Jurisprudenz ihr Wissen zusammenfassend präsentiert, tut sie das als "Lehrbuch«; wohingegen die Soziologie es hier nur bis zum "Handbuch « (mit Überblicksartikeln) und zur "Einführung « schafft. Welche Profite bringt es, die Forschungsideale der Jurisprudenz auf das rechtssoziologische Forschungsgebiet anzuwenden? Klarheit, Konzentration und Verkleinerung - wie es scheint. Die Fragestellungen werden transparent, sie werden knapp gefaßt, und sie verlieren offenbar gerade dadurch - an Assoziationenreichtum. Der Gewinn für die Rezeption wird durch eine planmäßige Armut an Ambiguität erkauft; aus Wildwuchs wird Bonsai.

Wahrscheinlich liegt darin das Prinzip eines jeden Lehrbuchs. Die hier besprochenen Exemplare werden durch derartige Feststellungen also gar nicht kritisiert. Sie leisten, was von ihnen erwartet wird: sie vermitteln reproduzierbares und memorierbares Wissen. Und da integre Rechtswissenschaftler die Feder führen, wird man zuverlässig über den ,Sach- und Streitstand informiert, bis hin zur diskreten Andeutung der 'herrschenden Meinung` (beispielsweise bei Röhl, S. 127).

Wie macht man nun Lehrbuchwissenschaft? Indem man prinzipiell yon diesen Annahmen ausgeht: Die Summe der vorhandenen Texte ist in hohem Maße erkenntnishaltig (Wahrheit); vieles darin bezieht sich aufeinander (Kumulation); Sachfragen gehen den Paradigmastreitigkeiten voran (Praxisbezug). Das Lehrbuch schreibt sich alsdann als übersichtlich geordnetes Kompendium von handlichen Begriffsklärungen und anwendbaren Forschungsresultaten.

Es macht die Eigenart der Soziologie aus, daß ihre Vertreter/innen dazu entweder sich zu fein oder zu skrupulös sind. Das Problematisieren ist ihnen wichtiger als das Praktischwerden. Wie sich beides verbinden läßt, führen die Juristen zwanglos vor.

Wer in ihren Lehrbüchern liest, soll sich vorerst die Lektüre der Ursprungsliteratur ersparen können. Dazu werden die sozialwissenschaftlichen Theoreme und Konzepte grundrißartig dargestellt. Röhl hat sogar ein Kapitel, welches die "Methoden empirischer Sozialforschung vermittelt (S. Io5-I I 8 ). Die Darstellungen sind, wie schon wiederholt betont, durchweg vertrauenswürdig; es zeichnet sie aus, was alle 
Welt als Professionalität von Juristen bewundert: ganze Literaturmassen und wahre Streitdschungel werden durchlichtet, werden sauf den Punkt gebrachte. Welchen Punkt nur? Nun, den der ,Relevanz: was Juristen an soziologischen Konstrukten interessieren könnte, wo die Sprachspiele einander berühren.

$\mathrm{Ob}$ man danach das Recht besser oder anders verstehen kann? Das ist die Frage nach dem Wert der Rechtssoziologie und zugleich nach dem Wert der besprochenen Bücher. Die Frage ist hinsichtlich der Buchautoren mit Nachdruck zu bejahen, hinsichtlich ihrer Leser/innen allerdings nur dann, wenn sie die Bücher wirklich lesen, anstatt sich bloß argumentative Versatzstücke herauszuklauben.

Das Konstruktionsprinzip juristischer Lehrbücher bringt ein scheinbar geschlossenes, ja organisches Korpus von Fragestellungen und Frageresultaten hervor ('Fälle mit Lösungen`, sozusagen). Unbeantwortbares wird an die Rechtsphilosophie weitergereicht. Hieraus sprechen Zuversicht in die Wissensproduktion und Vertrauen auf die Loyalität des soziologischen Erkennens, die dort nur schlechten Gewissens registriert werden können, denen aber nicht entsprochen werden kann.

Sozialwissenschaftliche Bücher, zumal wenn nicht von abtrünnigen Jüngern jurisprudentiae verfaßt, neigen dazu, vorhandene Antworten in Frage zu stellen. Sie trauen den Resultaten nicht, sei nun die Theorie zu simpel, sei die Gesellschaft längst woanders. Rottleuthners Buch steckt voller Exempel dafür, wie jeder noch so gelungene Forschungsbericht ein Mehrfaches neuer Forschungsprobleme sehen läßt. Dem dezisiven Erkenntnisideal der Jurisprudenz steht das reflexive der Soziologie nahezu diametral entgegen. Eine Rechtssoziologie, welche die Jurisprudenz nicht irritierte, wäre keine mehr.

Entgegen der Meinung unserer juristischen Rechtssoziologen ist die Philosophee nicht ein zwar benachbartes, aber abgetrenntes Gelände. Vielmehr, so meine ich, bewohnt sie dasselbe Haus wie die Soziologie, und man trifft sich allenthalben auf den Fluren. Wenn Juristen die Rechtssoziologie von der Rechtsphilosophie säuberlich abtrennen (siehe Raiser, S. rof.; Rehbinder, S. 1; vgl a. Röhl, S. 5, 372), dann wollen sie Soziologie vor mancherlei Verdacht schützen, obwohl es eine Mohrenwäsche bleibt. Manche dieser Autoren betreiben überdies Soziologie und Philosophie (oder 'Theories) des Rechts in Personalunion, qua Lehrstuhl oder Parallelveröffentlichung. Am Verhältnis zwischen Soziologie und Philosophie interessieren die Kontakte und Bezüge, kaum aber die Unterschiede.

$X$.

Meine Bewunderung für die immense Leistung der Autoren paart sich mit einer Verstörung. Den Lehrbüchern der Juristen gelingt, was in der Soziologie als unmöglich gilt: das Forschungsgebiet der (Rechts-)Soziologie übersichtlich zu ordnen, den Stoff ohne Reste zu systematisieren. Das Resultat ist eine rationalisierte Gesellschaftswissenschaft. Noch das notorisch Unordentliche an Gesellschaft und Soziologie erhält seinen Platz; es verschwindet in Kapitelchen über Konflikt und Wandel. Vorgänge von Revolution, Subkultur kommen kaum vor. Erst recht stehen Gewalt, Trieb u. ä. in einer anderen, der Vernunft abgewandten Welt (vgl. etwa Raiser, S. 340).

Die Reinigung macht einen Gutteil der eindrucksvollen Geschlossenheit der Darstellungen aus, verantwortet aber auch eine gewisse Sterilität. Wie sich Gesellschaft, Recht und Soziologie weiterentwickeln, wird von anderen Impulsen ausgehen als sie in dieser Art von Literatur angesprochen sind resp. sein können.

Die farbigen, die dunklen und die paradoxen Seiten von Recht und Gesellschaft sind Sache dieser Autoren nicht. Doch geradewegs von hierher, aus den Gegentendenzen 
des scheinbar Begriffenen, speisen sich die Entwicklungen im Sozialen und im

Erkennen. Selbstverständlich schickt sich solches nicht für ein Lehrbuch, das gattungsgemäß das nach- und abprüfbare Wissen kodifiziert. Soziologie als Erkenntnis aber ist tatsächlich dazu angetreten, hinter den Spiegel zu blicken und Horizonte zu überschreiten. In Flaschen abgefüllt und konserviert verliert sich das Aroma. Für die Rechtssoziologie bedeutet das allerdings nicht bloß eine Frage des Geschmacks - es könnte eine der Existenz sein.

\section{Sibylle Tönnies Eines Nachmittags im Juristischen Seminar}

Ducken muß man sich, bevor man in die Juristenfabrik hineinkommt. Keine Eingangshalle, sondern ein enger Betonschlauch nimmt einen auf, der zu einem Nadelöhr führt, durch das man schlüpfen muß, bevor man sich in der Welt der juristischen Literatur tummeln darf. Das Nadelöhr besteht aus einer durch Stellwände erreichten Engführung, die wie ein Kleinlabyrinth um einige Ecken führt, herum um einen Glaskasten, in dem ein Kontrolleur den Ein- und Ausgang beobachtet, den Ausgang daraufhin, ob nicht vielleicht ein Baumbach-Hefermehl mitgeführt wird oder ein Schwarz-Kleinknecht, den Eingang daraufhin, ob nicht ein zu diesem Zweck bestimmtes Gefäß hereingetragen wird. Nun, man hat passiert und ist drin, drin in der Fabrikhalle mit unendlichen Regalreihen und unendlichen Tischreihen, an denen mit gebeugten Rücken die Juristen der Zukunft ihre Nasen in die Baumbach-Hefermehls und Schwarz-Kleinknechts stecken. Wenn sie aber aufstehen und zwischen den Regalen flanieren, sind sie keineswegs gebeugt, sondern flotte, frischgeschorene, frischgekämmte, frischgewaschene Yuppies in guten Jeans und Markenpullovern.

Ja, und dazwischen bewegt sich die nicht mehr ganz junge Dame und wundert sich, wie fremd ihr diese Welt geworden ist.

Die nicht mehr ganz junge Dame bin ich natürlich selbst. Nach einigen Stunden Arbeit, einigen Stunden Hin- und Herlaufen zwischen den Bücherreihen und den Räumen, treppauf treppab durch die Etagen verliert sich das Gefühl des Fremdseins. Unten im Magazin riecht es wie vor fünfundzwanzig Jahren schon, als das Juristische Seminar noch in einem anderen Gebäude lag, im Hochhaus 4.-7. Stock, wo ich studiert und Stein-Jonas und Thomas-Putzo kennengelernt habe. Die tausend und abertausend verstaubten Papiere haben ihren Geruch mitgenommen in das neue Betonhaus hinten am Autobahnzubringer. Dieser Geruch, den ich länger kenne als die anderen hier im Gebäude, gibt mir das Gefühl, hier doch nicht so verkehrt zu sein, hier doch auch hinzugehören - in gewisser Weise hier besonders hinzugehören. Nach einigen Stunden bewege ich mich frei in diesen Hallen und fühle mich auch nicht mehr älter als die jungen Leute, spüre im Gegenteil in meinen Bewegungen eine provozierende Jugendkraft, spüre, wie die Unternehmungslust, mit der ich meine Bücher suche, hier fehl am Platz ist, - spüre die Jakobinermütze, die mir am Kopf festgewachsen ist und die jetzt offenbar rot leuchtet.

$\mathrm{Da}$ ist doch noch jemand in diesem Gebäude, der mit dem Staubgeruch umgezogen ist, der mit allen Thomas-Putzos und Baumbach-Hefermehls eingepackt und umgeladen worden ist - Professor X. Er wandelt jovial auf den Gängen, gealtert, aber keineswegs verfallen, im Gegenteil gestrafft und gestärkt, nicht mehr der unsicher- 